\title{
Spectral properties of INTEGRAL Gamma-Ray Bursts
}

\author{
G. Vianello* \\ INAF - IASF Milano, Via Bassini 15, I-20133 Milano, Italy \\ Dip. di Fisica e Matematica, Università dell'Insubria, via Valleggio 11, I-22100 Como, Italy \\ E-mail: vianellodiasf-milano.inaf.it \\ D. Götz \\ CEA-Saclay, DSM/Irfu/Service d'Astrophysique, Orme des Merisiers, F-91191, Gif-sur-Yvette, \\ France
}

\section{S. Mereghetti}

INAF - IASF Milano, Via Bassini 15, I-20133 Milano, Italy

\begin{abstract}
We present the global properties of the bursts detected and localized by the IBIS instrument on the INTEGRAL satellite from November 2002 to September 2008. This sample is now composed of 56 bursts, corresponding to a rate of $\sim 0.8 \mathrm{GRB}$ per month. Thanks to the performances of the Integral Burst Alert System, 50\% of the IBIS GRBs have detected afterglows, while 5\% have redshift measurements. We have carried out a spectral analysis of the 43 bursts in the INTEGRAL public archive, using the most recent software and calibration. We have derived an updated, homogenous and accurate catalogue with the spectral features of the sample (Vianello et al., 2008 , in preparation). While most of the spectra are well fitted by a power law with photon index $\sim 1.6$, we found that 9 bursts are better described by a cut-off power law. These results are comparable with those obtained with BAT onboard Swift. There is a marginal evidence that ISGRI detects dimmer bursts than Swift/BAT. Using the revised spectral parameters and an updated sky exposure map that takes into account also the effects of the GRB trigger efficiency, we strengthen the evidence for a spatial correlation with the super galactic plane of the faint bursts with long spectral lag reported by Foley et al. (2008).
\end{abstract}

7th INTEGRAL Workshop

September 8-112008

Copenhagen, Denmark

\footnotetext{
${ }^{*}$ Speaker.
} 


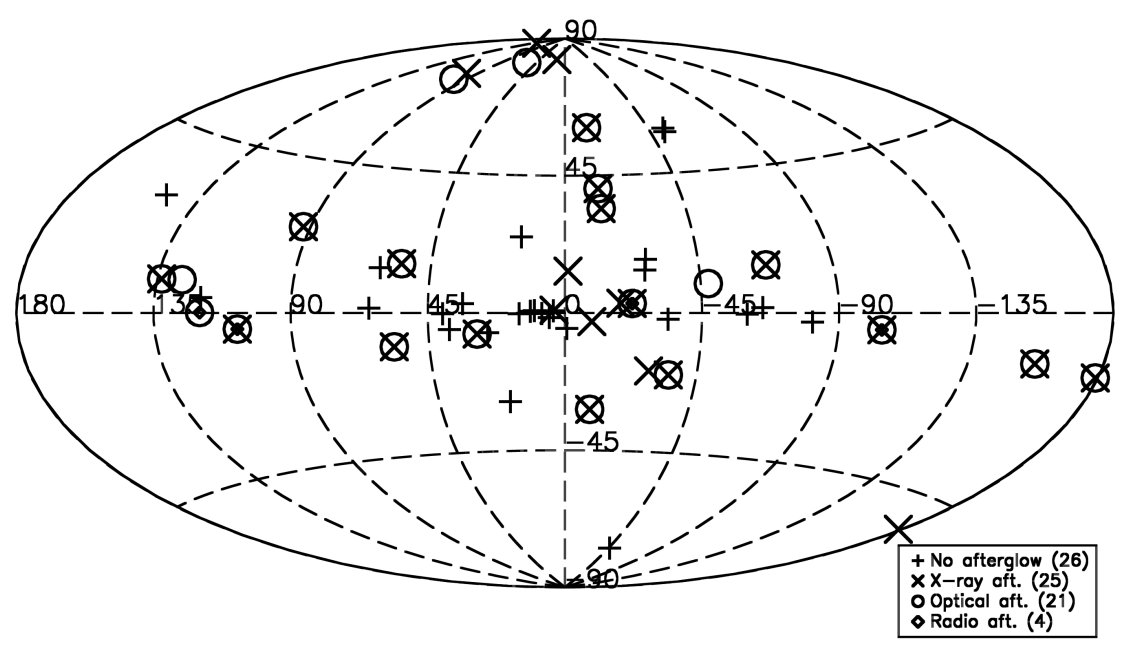

Figure 1: Sky positions of the GRBs detected by IBIS in Galactic coordinates.

\section{Introduction}

The INTEGRAL satellite (1), launched in October 2002, carries a set of coded mask instruments dedicated to fine imaging and spectroscopy in the soft $\gamma$-ray energy domain (15 keV-10 $\mathrm{MeV}$ ). Even if INTEGRAL is not a mission specifically dedicated to GRBs, its imager instrument IBIS (2) is well suited for GRBs studies, in particular with its low energy detector plane ISGRI (15 keV-1 MeV) (3). Pre-launch expectations of detecting about one GRB per month in the IBIS field of view prompted the development of the INTEGRAL Burst Alert System (IBAS). IBAS (4) is a software system, running on ground at the INTEGRAL Science Data Centre (ISDC; 5), able to detect and localize in real time the GRBs in the IBIS field of view. Thanks to IBAS, the INTEGRAL satellite has been the first one to provide GRB positions with an uncertainty of only $\sim 2-3$ arcmin in near real time (less than a few tens of second after the burst trigger). To date 56 bursts have been detected in IBIS/ISGRI data ${ }^{1}$. We are building up an updated catalog of all the GRBs localized with INTEGRAL, containing a detailed spectral analysis of the bursts for which public data are available. The main purpose of our work is to provide a homogeneous set of results, obtained with the latest instrument calibration and software. In several cases the spectral parameters we derived differ from, and supersede, those reported in previous analysis, that were based on earlier software versions and calibrations. Here we summarize some of the preliminar results we have obtained, and refer to (6, in preparation) for other results and for all the details about the analysis procedures.

\section{The IBIS coded-mask telescope}

IBIS (2) is an imaging gamma-ray telescope, based on the coded-mask technique, with a square field of view of $29^{\circ} \times 29^{\circ}$ (at zero sensitivity). The sensitivity is maximum and nearly uniform within the inner $8.5^{\circ} \times 8.5^{\circ}$, corresponding to the so called fully coded field of view. Our analysis is mainly based on data taken with the IBIS low energy detector ISGRI (3). The

\footnotetext{
${ }^{1}$ see http://ibas.iasf-milano.inaf.it
} 


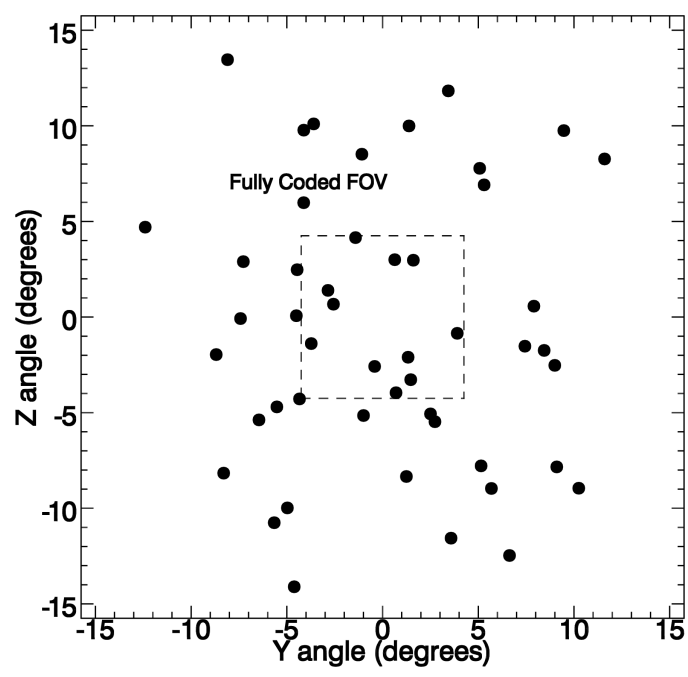

Figure 2: GRBs positions in the detector coordinates. The region inside the dotted rectangle is the fully coded field of view.

CdTe detector thickness of $2 \mathrm{~mm}$ ensures an efficiency of $50 \%$ at $150 \mathrm{keV}$. Above this energy the efficiency decreases rapidly (10\% at $300 \mathrm{keV})$, and although the brightest GRBs are detected up to $\sim 500 \mathrm{keV}$, the majority of the spectra cover the $18-300 \mathrm{keV}$ energy range .

\section{GRB sample}

ISGRI observed 56 GRBs up to the end of September, 2008. The sky distribution of the bursts is plotted in galactic coordinates in Fig. 1. The concentration of events close to the galactic plane simply reflects the larger exposure devoted to these regions by the pointing program of INTEGRAL . Most of the bursts were discovered and localized with the INTEGRAL Burst Alert System (IBAS; 4) and their coordinates were automatically distributed in near real time within a few seconds. The remaining ones were detected during the initial IBAS performance verification phase, in offline searches, or after interactive verification of low significance IBAS internal alerts. The IBAS trigger efficiency is maximum in the fully coded field of view, while decreases going at larger offaxis angle, so that we need a brighter burst to obtain the same signal-to-noise. On the other hand the sky area covered by the detector increases with the off-axis angle. Thus it is not surprising that the majority of GRBs have been detected at intermediate off-axis angles, as visible in Fig. 2 where we show the instrumental positions of the bursts. Some GRBs were detected at the very edge of the field of view, showing that the IBAS system is able to detect bursts in the whole field of view, provided the signal to noise is large enough. The errors on the coordinates provided by IBAS are in most cases between 1.5 and 3 arcmin, basically limited by the statistical uncertainties. The difference between the ISGRI and the afterglow position, when available, is compared with the quoted uncertainties in Fig. 3. All the positions of the afterglows are within the error regions provided by ISGRI, thus the localization of GRBs are accurate and the error radii are correctly estimated. Moreover there is no evidence of systematic errors depending on the position of the burst on the detector. 


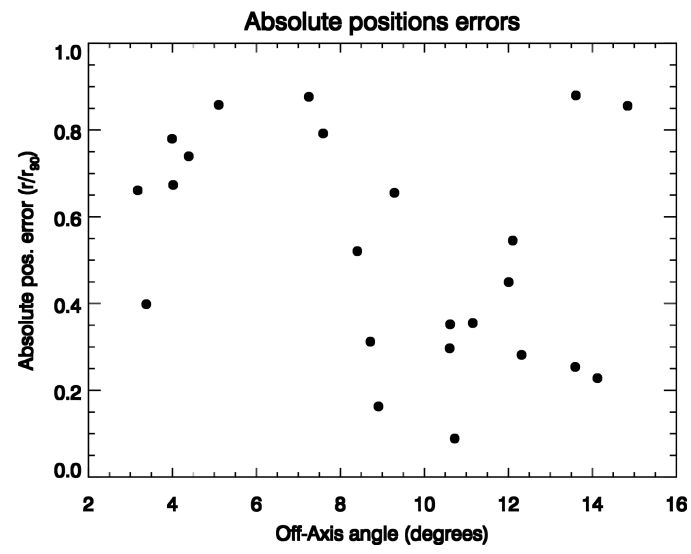

Figure 3: Absolute position errors $r$ as a function of the off-axis angle, for bursts with a detected afterglow. $r$ is defined as the difference between the position obtained with ISGRI data and that of the afterglow. The absolute position error is given in units of $r_{90}$, that is the 90 percent cl. error radius estimated during the analysis of ISGRI data. All the afterglows were found inside the error circle provided by ISGRI.

\section{Data analysis}

\subsection{Light curves extraction}

To extract the GRB light curves, as well as for most of our timing analysis, we used the software tools developed for the IBAS interactive quick-look analysis. These tools are more suitable than the standard OSA software for this kind of analysis. Some examples of the background subtracted light curves, in the $15-300 \mathrm{keV}$ range, are shown in Fig. 4. The light curves have different bin sizes, appropriate to the counts statistics, and the plotted count rates are normalized taking into account the detector fraction illuminated by the GRBs at the different off-axis angles. All the light curves are corrected for the instrumental dead time, that increased from $\sim 20 \%$ at the beginning of the mission to the current value of $\sim 30 \%$. Some gaps due to telemetry saturation occurring during the brightest parts of the bursts are visible in the light curves. We have used the light curve of every GRB to determine its $t_{90}$, the duration over which $90 \%$ of the fluence of the burst is observed. These intervals are indicated by the dashed lines in Fig. 4. In Fig.5 we report the distribution of the durations.

\subsection{Spectral extraction and models}

We consider for the spectral analysis only the bursts observed until March of 2007, since the data of the remaining ones are not yet publicly available. For the spectral analysis we use the latest release of the standard software (OSA 7.0) distributed by the ISDC. The time integrated spectra of the faintest bursts are extracted from the $t_{90}$ time intervals. In the other cases, the larger statistics allow us to use longer time intervals, indicated by the dotted lines in Fig. 4. We consider the following spectral models:

- power law (PL) with photon index $\alpha$ :

$$
f(E)=A E^{-\alpha}
$$



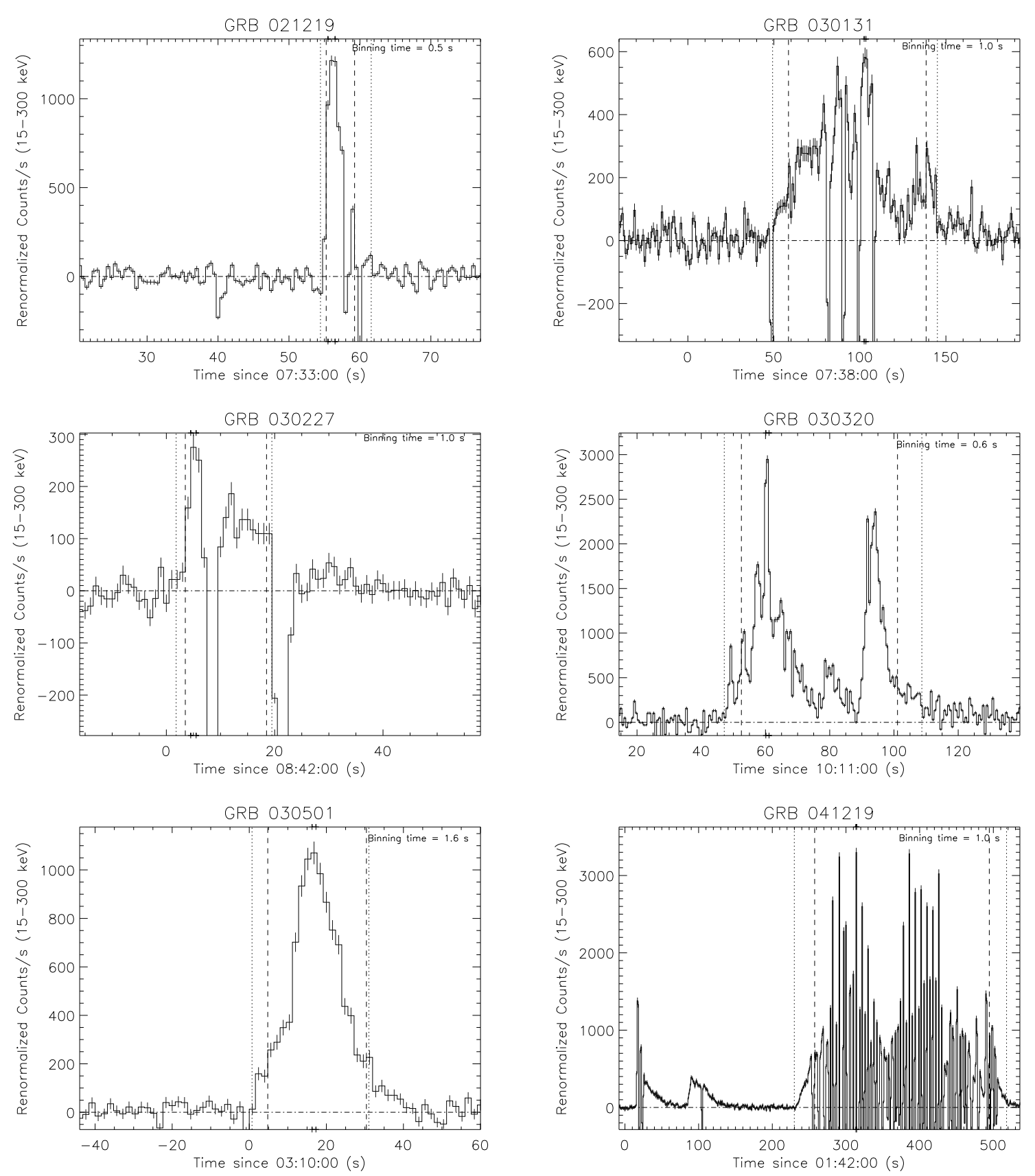

Figure 4: Light curves of some GRBs detected by ISGRI.

- power law with exponential cutoff (CPL):

$$
f(E)=A E^{-\alpha} e^{-E / E_{0}}
$$

- Quasi-thermal model (QT) $(7 ; 8 ; 9)$, consisting of a power law and a blackbody:

$$
f(E)=A E^{-\alpha}+B \frac{E^{2}}{(k T)^{4}\left(e^{\frac{E}{k T}}-1\right)}
$$




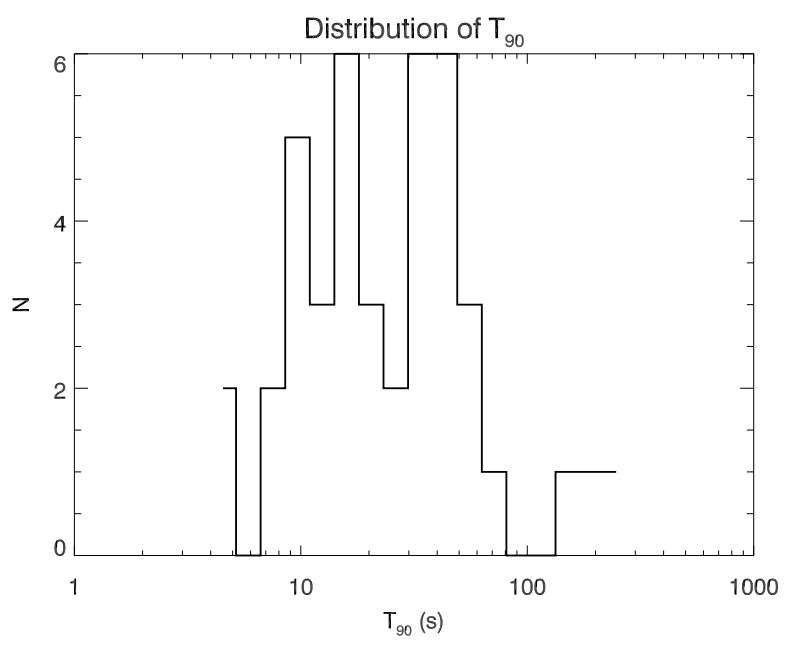

Figure 5: Distribution of $t_{90}$ for INTEGRAL GRBs we have analized in this work. No short bursts $\left(t_{90}<2\right.$ s) are present in the sample.

We also try to fit the spectra of bright bursts using the Band model, consisting of two power laws joined with continuity (10), which is widely used to fit GRB spectra. However, these fits to our ISGRI data could not constrain the values of the high-energy photon index. This means in practice that the Band model reduces to the CPL model when used in a energy band as small as ours.

\section{Results}

\subsection{Spectral results}

The spectra of INTEGRAL GRBs are generally well described by the powerlaw model, with photon index values around $\sim 1.6$ (see Fig. 6). Most of the bursts spectra poorly fitted with this model give acceptable fits with the introduction of an exponential cut-off. Almost all the bursts showing evidence for a curved spectrum in the ISGRI energy range can also be fitted with the QT model.

We have measured the cutoff energy $\mathrm{E}_{0}$ in 9 out of $43 \mathrm{GRBs}$, corresponding to $\sim 20 \%$ of our sample. The values of $\mathrm{E}_{0}$ are in the range $50-150 \mathrm{keV}$, and clustered around $80 \mathrm{keV}$ (see Fig. 7).

This is not surprising, because most of the spectra have a significant signal from $18 \mathrm{keV}$ to 300 $\mathrm{keV}$, but the spectral bins above $200 \mathrm{keV}$ usually have large statistical errors. In order to constrain the cutoff energy, a significant number of energy bins with good statistics are needed both below and above the cutoff value. Thus, the limited bandpass of ISGRI introduces a bias in the cutoff measurement, as also seen in similar instruments, like Swift/BAT (see i.e. 11). (12) studied a large sample of BAT bursts detecting a cutoff energy in 32 long GRBs out of 216 , a percentage $(\sim 15 \%)$ even smaller than that found in our sample.

The majority of GRBs observed in the past are well fitted by the Band model (10; 13). Analyzing the subsample of very bright GRBs, (14) (hereinafter K06) found that the average values for the low- and high-energy photon index are $\alpha \simeq 1.1$ and $\beta \simeq 2.3$, respectively. The two photon index 


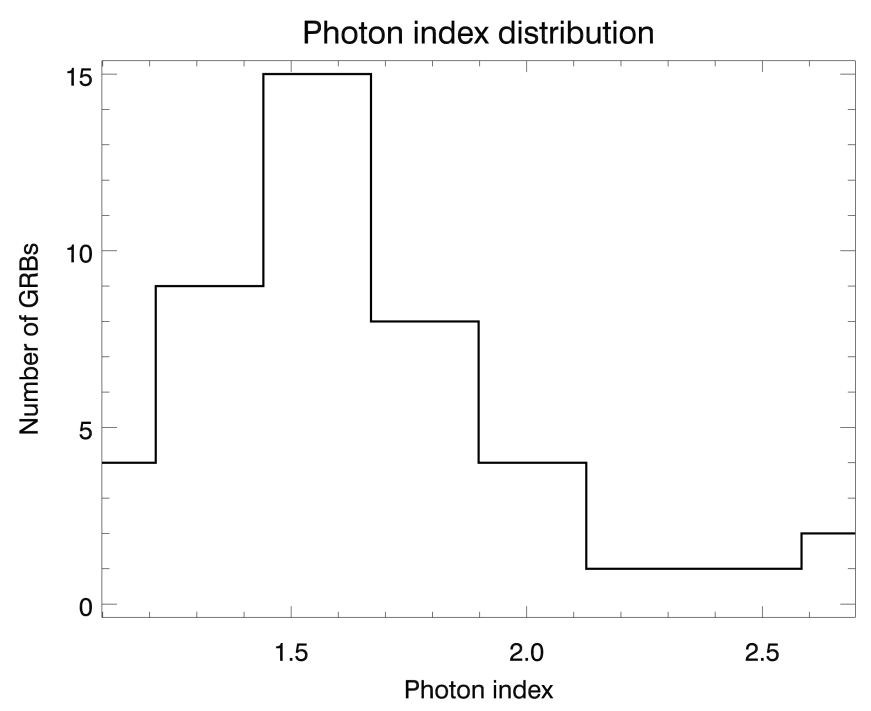

Figure 6: The distribution of photon index of the single power law fits. All but two spectra can be well described by a power law and are comprised in this plot, altough for 9 of them a CPL or a QT model give a better fit.

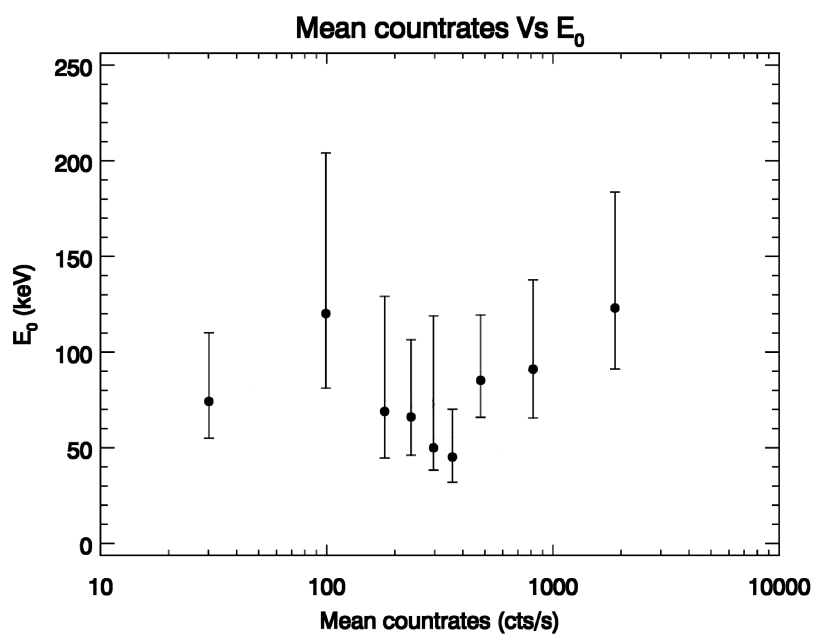

Figure 7: Cut-off energy $E_{0}$ as a function of the mean countrate (background subtracted, not renormalized) measured during each GRBs. The error bars are at the $90 \%$ confidence level.

distributions are clustered around these values and well separated. Our photon index distribution (Fig. 6) peaks around 1.6, a value between the average values of $\alpha$ and $\beta$. A similar result was found for Swift/BAT GRBs (12). If the results found by K06 using only the brightest bursts apply to the whole GRB population, we can conclude that we are unable to see some cutoff energies either because they are within the ISGRI energy range but the spectra do not have enough statistics, or because the cut-off lies outside the ISGRI energy band. In both cases the observed spectrum would have some curvature, so that in both cases the best fit with a single power law would give a photon index intermediate between $\alpha$ and $\beta$. 

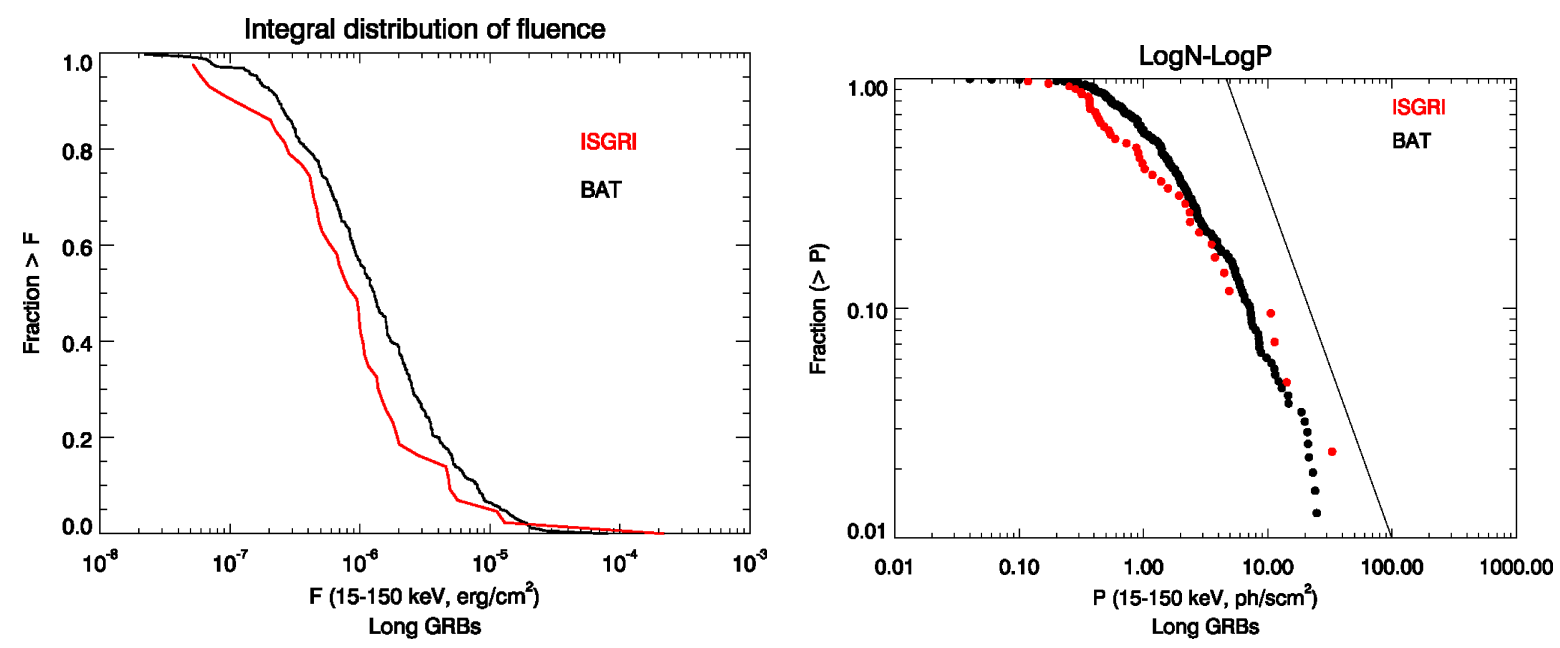

Figure 8: Normalized integral distributions of fluences (left) and peak fluxes (right) for both ISGRI and BAT.

\subsection{Spectral evolution}

For 7 GRBs the detected fluence was high enough to perform time-resolved spectral analysis. 6 bursts out of 7 have shown different kinds of spectral evolution. We can divide the spectrally evolving bursts in two classes: single-peaked bursts with a spectral evolution, and multi-peaked bursts with spectral differences between the peaks. In the first class we detected only one case of the classical hard-to-soft evolution. In the second class we detected two cases of hard-to-soft evolution, one case of soft-to-hard evolution and two cases of more complex evolution. In one case we have detected no evolution at all between the peaks.

\subsection{Peak fluxes distribution}

In Fig. 8 we show the normalized integral distributions of fluences and peak fluxes for both ISGRI and BAT. We expect the burst populations seen by the two instruments to be very similar, because the two instruments are very similar. If we apply a Kolmogorov-Smirnoff (KS) test to the data of Fig. 8, we obtain a probability of $12 \%$ that the ISGRI and BAT fluences are drawn from the same distribution, while for the peak fluxes the probability is $3 \%$. The latter result suggests that the two populations could be somewhat different, with ISGRI detecting fainter GRBs than BAT. However, larger samples are required to eventually confirm this possible difference.

\subsection{The rate of short bursts}

The BATSE experiment onboard CGRO found that $\sim 25 \%$ of GRBs are short hard bursts. ISGRI detected instead only 2 short GRBs out of 56 bursts, corresponding to $\sim 3.5 \%$. Moreover, Swift/BAT found $\sim 8 \%$. The BATSE experiment, more sensitive at higher energy than both ISGRI and BAT, was better suited to detect short hard bursts. This could easily explain the different percentage of short GRBs between BATSE and both ISGRI and BAT. The differences between two very similar instrument like ISGRI and BAT are harder to explain. However we note that 
the sample of ISGRI GRBs is quite small, thus affected by large statistical uncertainties. If we hypotize that the trigger efficiency is the same between the two instruments, the measure of ISGRI is indeed marginally consistent with that of BAT. This discrepancy could vanish as the number of bursts detected by ISGRI increases, but it is certainly a point to be adressed in the future.

\subsection{Spatial Distribution}

While the whole INTEGRAL GRB sample is isotropically distributed, the sub-sample of 10 bursts with a spectral $\operatorname{lag}^{2} \tau>0.75 \mathrm{~s}$ and low luminosity is spatially correlated with the supergalactic plane (15). This result, which supports the existence of a local GRB population, relies on an accurate estimate of the INTEGRAL sky coverage. In computing the sky coverage, those authors used the INTEGRAL exposure maps, but neglected other factors affecting the sensitivity of GRB triggers. Taking into account this and other similar effects we have enhanced the statistical significance of the detected anisotropy from $\sim 2.5$ to $\sim 3 \sigma$.

\section{Conclusions}

Using the latest available software and calibration, we are deriving accurate spectral parameters for all the INTEGRAL bursts in a coherent way, superseding and harmonising all the results that could be found in literature before and adding the lacking ones. We are also able to confirm, with a higher significance, the clustering of faint, long-lag bursts around the supergalactic plane, supporting the existence of a local GRB population.

The INTEGRAL and Swift experience has shown that wide field coded mask instruments are a powerful tool for GRB science, but the lack of sensitivity over a broad bandpass hampers the progress. Even in presence of a redshift determination, with the simple power law description a key parameter like the overall energy budget of the sources cannot be determined, as well as any sensitive attempt to model the prompt GRB emission cannot be performed. Future GRB missions should carry adequate instruments in order to obtain prompt localizations and broad band spectroscopy at once.

\footnotetext{
${ }^{2}$ The spectral lag $\tau$ is the time difference between the position of a peak as seen in two light curves extracted from different energy bands.
} 


\section{References}

[1] C. Winkler, T. J.-L. Courvoisier, G. Di Cocco, N. Gehrels, A. Giménez, S. Grebenev, W. Hermsen, J. M. Mas-Hesse, F. Lebrun, N. Lund, G. G. C. Palumbo, J. Paul, J.-P. Roques, H. Schnopper, V. Schönfelder, R. Sunyaev, B. Teegarden, P. Ubertini, G. Vedrenne, and A. J. Dean, The INTEGRAL mission, A\&A 411 (Nov., 2003) L1-L6.

[2] P. Ubertini, F. Lebrun, G. Di Cocco, A. Bazzano, A. J. Bird, K. Broenstad, A. Goldwurm, G. La Rosa, C. Labanti, P. Laurent, I. F. Mirabel, E. M. Quadrini, B. Ramsey, V. Reglero, L. Sabau, B. Sacco, R. Staubert, L. Vigroux, M. C. Weisskopf, and A. A. Zdziarski, IBIS: The Imager on-board INTEGRAL, A\&A 411 (Nov., 2003) L131-L139.

[3] F. Lebrun, J. P. Leray, P. Lavocat, J. Crétolle, M. Arquès, C. Blondel, C. Bonnin, A. Bouère, C. Cara, T. Chaleil, F. Daly, F. Desages, H. Dzitko, B. Horeau, P. Laurent, O. Limousin, F. Mathy, V. Mauguen, F. Meignier, F. Molinié, E. Poindron, M. Rouger, A. Sauvageon, and T. Tourrette, ISGRI: The INTEGRAL Soft Gamma-Ray Imager, A\&A 411 (Nov., 2003) L141-L148,'[arxivastro-ph/0310362];

[4] S. Mereghetti, D. Götz, J. Borkowski, R. Walter, and H. Pedersen, The INTEGRAL Burst

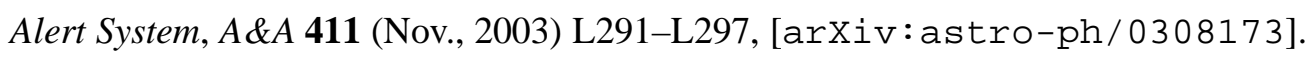

[5] T. J.-L. Courvoisier, R. Walter, V. Beckmann, A. J. Dean, P. Dubath, R. Hudec, P. Kretschmar, S. Mereghetti, T. Montmerle, N. Mowlavi, S. Paltani, A. Preite Martinez, N. Produit, R. Staubert, A. W. Strong, J.-P. Swings, N. J. Westergaard, N. White, C. Winkler, and A. A. Zdziarski, The INTEGRAL Science Data Centre (ISDC), A\&A 411 (Nov., 2003) L53-L57, Iärxìv:astrōph/0308047!

[6] G. Vianello, D. Götz, and S. Mereghetti, The updated spectral catalog of INTEGRAL Gamma-ray bursts, $\AA$ O00 (Dec., 2008).

[7] F. Ryde, Is Thermal Emission in Gamma-Ray Bursts Ubiquitous?, ApJ 625 (June, 2005) L95-L98, [arXiv:astro-ph

[8] C. Thompson, P. Mészáros, and M. J. Rees, Thermalization in Relativistic Outflows and the Correlation between Spectral Hardness and Apparent Luminosity in Gamma-Ray Bursts, ApJ 666 (Sept., 2007) 1012-1023, '[arXiv:astro-ph/0608282].

[9] G. Ghirlanda, Z. Bosnjak, G. Ghisellini, F. Tavecchio, and C. Firmani, Blackbody components in gamma-ray bursts spectra?, MNRAS 379 (July, 2007) 73-85, [arXiv:070 0 - 3438$]$

[10] D. Band, J. Matteson, L. Ford, B. Schaefer, D. Palmer, B. Teegarden, T. Cline, M. Briggs, W. Paciesas, G. Pendleton, G. Fishman, C. Kouveliotou, C. Meegan, R. Wilson, and P. Lestrade, BATSE observations of gamma-ray burst spectra. I - Spectral diversity, ApJ $\mathbf{4 1 3}$ (Aug., 1993) 281-292. 
[11] G. Ghirlanda, L. Nava, G. Ghisellini, C. Firmani, and J. I. Cabrera, The Epeak-Eiso plane of long gamma-ray bursts and selection effects, MNRAS 387 (June, 2008) 319-330, [arXiv:0804.1675];

[12] T. Sakamoto, S. D. Barthelmy, L. Barbier, J. R. Cummings, E. E. Fenimore, N. Gehrels, D. Hullinger, H. A. Krimm, C. B. Markwardt, D. M. Palmer, A. M. Parsons, G. Sato, M. Stamatikos, J. Tueller, T. N. Ukwatta, and B. Zhang, The First Swift BAT Gamma-Ray Burst Catalog, ApJS 175 (Mar., 2008) 179-190, [0 707.4626 ].

[13] R. D. Preece, M. S. Briggs, R. S. Mallozzi, G. N. Pendleton, W. S. Paciesas, and D. L. Band, The BATSE Gamma-Ray Burst Spectral Catalog. I. High Time Resolution Spectroscopy of Bright Bursts Using High Energy Resolution Data, ApJS 126 (Jan., 2000) 19-36,

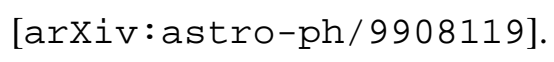

[14] Y. Kaneko, R. Preece, M. Briggs, W. Paciesas, C. Meegan, and D. Band, The Complete Spectral Catalog of Bright BATSE Gamma-Ray Bursts, ApJ Suppl. 166 (Sept., 2006) 298-340, '[arXiv:astro-ph/0601188]'.

[15] S. Foley, S. McGlynn, L. Hanlon, S. McBreen, and B. McBreen, Global characteristics of GRBs observed with INTEGRAL and the inferred large population of low-luminosity GRBs, A\&A 484 (June, 2008) 143-157, '[arXiv:0803.1821] 